Gynäk. Rdsch. 1967;4:199

\title{
Sehstörungen nach Gabe von Antigest
}

\begin{tabular}{|l|l|}
\hline K. & Dvořák \\
\hline M. & Arenberger \\
\hline
\end{tabular}

Institut für Ärztliche Fortbildung, Frauenklinik (Dlrektor: Doz. Dr. A. Černoch) und I. Universitäts-Augenklinik der Karls-Universität (Direktor: Prof. Dr. E. Dienstbier), Prag

Sehstörungen zählen zu den seltenen Komplikationen nach der Gabe peroraler Antikonzeptiva. Die Autoren berichten ausführlich über drei Fälle von Sehstörungen die nach der Gabe von Antigest B bei einer Gruppe von 134 Frauen beobachtet wurden. Im Vordergrund der Symptoma-tik standen Sehschärfeverlust

Nebelsehen

Augenbrennen

Tränen-fluß

Lichtscheue

Farbringe und gelegentlich Lidödeme. In keinem der drei Fälle konnten bei wiederholten Kontrollen krankhafte Befunde an Auge oder Augenhintergrund erhoben werden. Alle subjektiven Beschwerden die nach unterschiedlich langer Ein-nahme der Präparate auftraten klangen nach Absetzen der Medika-tion sehr rasch ab.

* Originaltítel: Poruchy vidëní po Antigestu. 